\title{
Poorly Differentiated Neuroendocrine Lesion
}

National Cancer Institute

\section{Source}

National Cancer Institute. Poorly Differentiated Neuroendocrine Lesion. NCI Thesaurus.

Code C155863.

A neoplastic lesion with neuroendocrine differentiation and poorly differentiated histological features. 